\title{
Chapter IV
}

The importance of fine-scale, vertical profiles in characterising nematode community structure

Results presented as

Steyaert M, Vanaverbeke J, Vanreusel A, Barranguet C, Lucas C, Vincx M (2003) The importance of fine-scale, vertical profiles in characterising nematode community structure.

Estuar Coast Shelf Sci 56:1-14 


\section{Abstract}

In this study the spatial heterogeneity of the nematode community on an intertidal flat (the Molenplaat) in the Westerschelde estuary (SW Netherlands) was investigated. It was tested to what extent macroscale (km's) variability was more important than microscale (m's) variability. In addition the importance of vertical distribution profile in the sediment in explaining the horizontal macroscale variability was evaluated. Differences in structure of the community were analysed at a kilometre scale at three sites that differed in chemico-physical features. The differences in geochemical and physical conditions on a horizontal scale were reflected in species composition and trophic structure of the nematode communities, and to a much lesser extent in their total abundance and species diversity.

Detailed investigation of vertical depth profiles showed more pronounced differences between environmentally divergent sites. Sediment granulometry appears to be important in controlling the fauna in the upper sediment layers. At depths similar faunal assemblages were found irrespective of sediment granulometry, suggesting that other environmental features are more dominant.

Vertically, nematode species showed depth distributions that were indicative of sediment characteristics related to the site-specific hydrodynamic regime. Pronounced vertical segregation of nematode species was observed within sandy sediment under strong hydrodynamic and food-stressed conditions. A surface-dwelling nematode community of large predatory enoplids was separated from a deposit feeding xyalid-microlaimid community in deeper sediment layers (beneath $2 \mathrm{~cm}$ ). Causal factors for this segregation are thought to be species interactions, feeding strategies and/or physical disturbance. In the finest sediments, with high silt content, almost all nematode species were confined to the upper sediment layers $(1.5 \mathrm{~cm})$. A sharp decline in density and diversity with depth was observed. Key factors for this distribution pattern are possibly related with the limited oxygen penetration in surface layers and the occurrence of sulphide in deeper sediment layers. At intermediate hydrodynamic and granulometric conditions, a gradual shifting nematode community was observed with depth, with dominant nematode species maxima present at specific depth layers. 


\section{Introduction}

Knowledge of spatial patterns of benthic organisms and the scale of these patterns contribute to a better understanding of benthic community structure and functioning. Such information is often the best, if not the only way for assessing interspecific interactions, which to a considerable extent determine community structure. Comparisons between the spatial patterns of consumers and resources provide information on trophic interactions and the spatial scales at which these interactions occur (Pinckney \& Sandulli 1990, Sandulli \& Pinckney 1999).

On a vertical scale of centimetres, the effect of abiotic characteristics of sediments (e.g. oxygen, water content, proximity to surface) on community structure is as important as the other abiotic variables (such as salinity, sedimentological and geomorphological variables), which act on a horizontal scale of hundreds of metres. Moreover, the ecological understanding of the functioning of meiobenthic communities is enhanced by knowledge of animal vertical distribution (Soetaert et al. 1994). It has been shown that many nematode species exhibit a typical vertical distribution which often relates to a variety of biological, physical and chemical variables (Warwick \& Gee 1984, Giere 1993, Hendelberg \& Jensen 1993, Wetzel et al. 1995, Soetaert et al. 1994, Steyaert et al. 1999). It has been argued that the vertical segregation of species will reduce the number of (competitive or predative) interactions, and this could explain the very high number of species that coexist in a certain small patch (Joint et al. 1982).

The spatial patterns of temperate nematode communities on different horizontal scales have already been investigated extensively in different estuaries. Most of these studies related structural patterns of the nematode assemblages to environmental variables as sedimentary and latitudinal gradients, food resources, salinity, disturbances of different nature (e.g. Warwick \& Gee 1984, Soetaert et al. 1995, Li et al. 1997, Guo et al. 2001, Neilson \& Boag 2002, Tita et al. 2002). The spatial patterns of nematode communities are well documented in intertidal and subtidal zones of the Westerschelde. Soetaert et al. (1994) found maximum abundance of the majority of the species in the intertidal zone. Intertidal communities exhibited a welldeveloped community gradient with sediment depth, whereas the subtidal and channel communities showed distinct and in some cases distorted community patterns associated with large socio-economic pressure by dredging, pollution and consequently oxygen depletion.

This study deals with the spatial heterogeneity of nematode associations on a small intertidal, estuarine flat. Differences in structure of the communities were initially analysed in terms of 
depth-integrated characteristics for a high number (5) of replicate samples (collected at a m scale) at three geographically separated (at km scale) and chemico-physically diverging sites. In addition to the comparison of bulk characteristics, spatial differences in community structure are established by micro-scaled vertical profile analysis of the same community parameters. We examined whether changing environmental conditions over a small system like the Molenplaat are reflected in the vertical distribution pattern of nematodes species and result in shifting community characteristics with depth in the sediment.

\section{Study site}

The Molenplaat $\left(51^{\circ} 26 \mathrm{~N}, 3^{\circ} 57 \mathrm{E}\right)$ is a small intertidal flat $\left(2\right.$ to $\left.3 \mathrm{~km}^{2}\right)$, located in the turbid, nutrient-rich and heterotrophic Westerschelde estuary (Fig. 1). Salinity in this region of the estuary varies between 20 and 25 psu (Herman et al. 2000). The flat is characterised by a high diversity of sediment types over a small distance. The ecology of the tidal flat has been studied during the project 'Eco-metabolism of an estuarine tidal flat' (ECOFLAT), and detailed background information on pigment distributions (Barranguet et al. 1997, Lucas \& Holligan 1999), microphytobenthos production (Barranguet et al. 1998, Barranguet et al. 2000), photosynthetic activity (Kromkamp et al. 1998), microphytobenthos resuspension (Lucas et al. 2000), nematode feeding behaviour (Moens et al. 1999a, 2000, Hamels et al. 2001), nematode tidal migration (Steyaert et al. 2001) and microbenthic (Hamels et al. 1998) and macrofaunal (Herman et al. 2000) communities is available. 


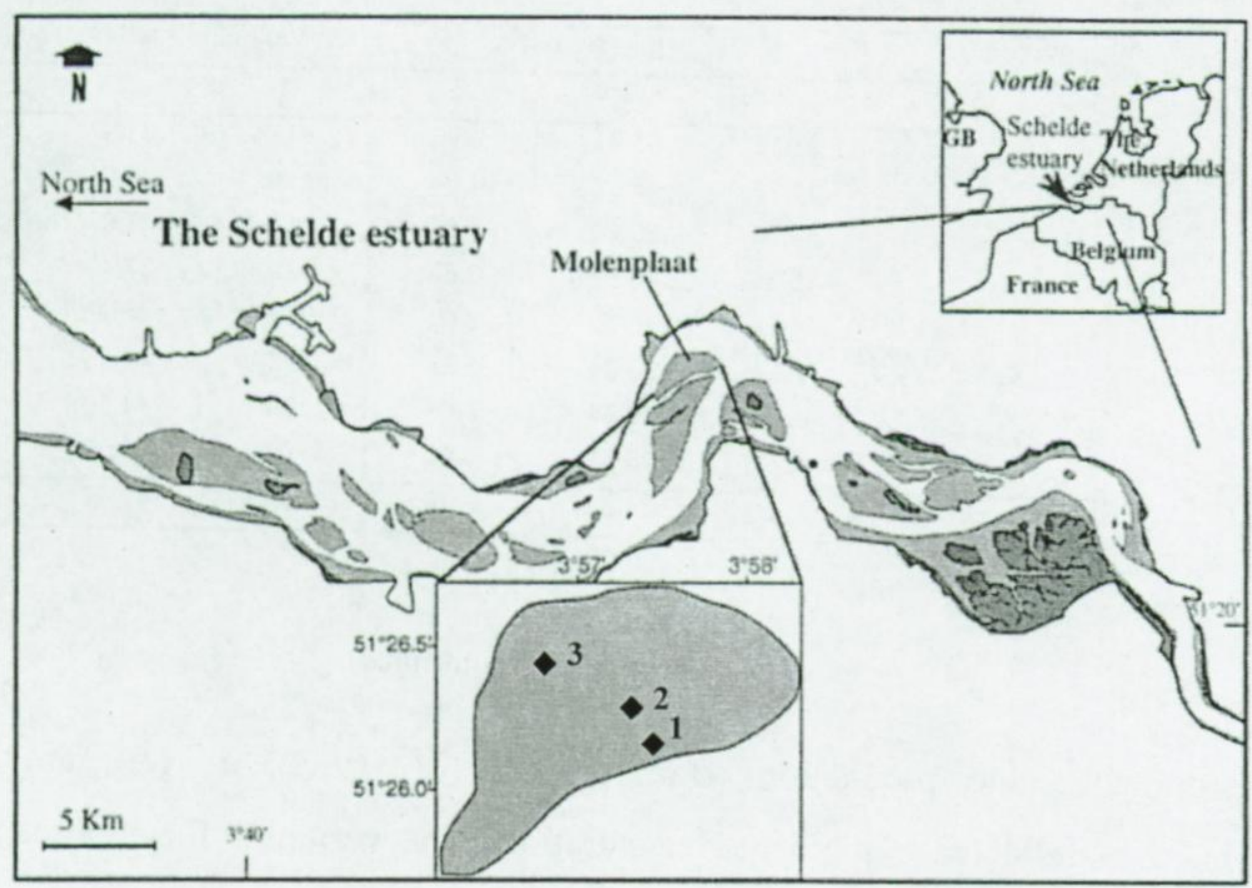

Figure 1. Location of the sampling sites on the Molenplaat

The three sites were selected on the basis of their sediment characteristics (Table 1). Site 2 $\left(57^{\circ} 2 \mathrm{~N}, 26^{\circ} 3 \mathrm{E}\right)$ has the finest sediment, Site $3\left(56^{\circ} 8 \mathrm{~N}, 26^{\circ} 4 \mathrm{E}\right)$ is more dynamic and sandier and Site $1\left(57^{\circ} 3 \mathrm{~N}, 26^{\circ} 15 \mathrm{E}\right)$ has intermediate characteristics (Herman et al. 2000, Widdows et al. 2000). Estimated of bottom shear stress (maximal value during a tidal cycle) were produced by a hydrodynamic model. Values are $0.36 \mathrm{~Pa}$ for Site 2, $0.43 \mathrm{~Pa}$ for Site 1 and 1.15 Pa for Site 3 (Van de Koppel et al. 2001). The average period of emersion varies between 4.5 $\mathrm{h}$ (Site 1$)$ and $7 \mathrm{~h}$ (Site 2 and 3 ) per tidal cycle.

\begin{tabular}{|l|ccc|ccc|ccc|}
\hline Depth $(\mathrm{cm})$ & \multicolumn{3}{|c|}{ Median grain size $(\mu \mathrm{m})$} & \multicolumn{3}{c|}{ Fine sand fraction $(\%)$} & \multicolumn{3}{c|}{ Medium sand fraction $(\%)$} \\
\hline Site & 1 & 2 & 3 & 1 & 2 & 3 & 1 & 2 & 3 \\
\hline $0-1$ & 136.79 & 76.95 & 169.58 & 49.31 & 24.46 & 80.71 & 6.13 & 0.55 & 6.97 \\
$1-2$ & 147.62 & 65.61 & 173.14 & 57.31 & 22.78 & 81.12 & 7.26 & 0.53 & 7.47 \\
$2-3$ & 127.63 & 44.81 & 173.14 & 43.77 & 12.37 & 79.18 & 6.87 & 0.40 & 8.46 \\
$3-4$ & 132.13 & 68.87 & 167.24 & 45.98 & 15.85 & 78.98 & 7.06 & 1.14 & 7.39 \\
$4-5$ & 132.13 & 0.01 & 173.14 & 46.61 & 1.57 & 77.87 & 6.56 & 0.04 & 9.33 \\
$5-6$ & 161.54 & 87.78 & 176.78 & 45.83 & 22.20 & 78.52 & 18.23 & 1.70 & 10.18 \\
$6-7$ & 192.11 & 95.39 & 171.94 & 48.11 & 27.71 & 77.18 & 24.94 & 0.75 & 9.17 \\
$7-8$ & 196.15 & 96.72 & 174.34 & 49.72 & 27.80 & 73.61 & 25.74 & 0.88 & 11.95 \\
\hline Mean & 153.26 & 67.02 & 172.41 & 48.33 & 19.34 & 78.40 & 12.85 & 0.75 & 8.87 \\
\hline
\end{tabular}

Table 1. Sediment characteristics for the three sites (data out of the ECOFLAT database, Herman et al. 2001) 


\begin{tabular}{|l|ccc|ccc|}
\hline Depth $(\mathrm{cm})$ & \multicolumn{3}{|c|}{ Silt (\%) } & \multicolumn{3}{c|}{ Very Fine sand fraction (\%) } \\
\hline Site & 1 & 2 & 3 & 1 & 2 & 3 \\
\hline $0-1$ & 24.13 & 43.32 & 3.96 & 19.74 & 31.67 & 8.14 \\
$1-2$ & 11.73 & 48.67 & 4.04 & no data & 28.01 & 7.20 \\
$2-3$ & 17.05 & 59.26 & 3.82 & 31.44 & 27.97 & 8.10 \\
$3-4$ & 15.35 & 45.51 & 3.88 & 30.74 & 37.49 & 9.42 \\
$4-5$ & 14.25 & 0.42 & 3.68 & 31.81 & no data & 8.47 \\
$5-6$ & 9.59 & 28.34 & 3.22 & 26.29 & 47.58 & no data \\
$6-7$ & 7.24 & 26.94 & 3.67 & 19.71 & 44.56 & 9.26 \\
$7-8$ & 6.71 & 26.61 & 4.03 & 17.82 & 44.66 & 9.15 \\
\hline Mean & 13.26 & 34.88 & 3.79 & 25.36 & 37.42 & 8.53 \\
\hline
\end{tabular}

Table 1. (continued)

Microphytobenthic production (Barranguet et al. 1998), as well as microphytobenthic biomass, as reflected in pigment concentrations, was very high for all three sites in June 1996 (Table 2).

\begin{tabular}{|l|ccc|ccc|ccc|}
\hline Depth (cm) & \multicolumn{3}{|c|}{ Chlorophyll a } & \multicolumn{3}{c|}{ Chlorophyll c } & \multicolumn{3}{c|}{ Fucoxanthin } \\
\hline Site & 1 & 2 & 3 & 1 & 2 & 3 & 1 & 2 & 3 \\
\hline $0-1$ & 939.39 & 175.20 & 16.48 & 123.77 & 22.95 & 2.96 & 465.62 & 140.34 & 8.55 \\
$1-2$ & 79.14 & 94.88 & 22.42 & 15.10 & 16.33 & 4.89 & 48.53 & 64.05 & 12.63 \\
$2-3$ & 130.06 & 50.14 & 10.03 & 23.53 & 7.18 & 2.77 & 68.46 & 30.92 & 7.90 \\
$3-4$ & 195.93 & 25.96 & 6.29 & 34.79 & 2.48 & 1.12 & 93.23 & 14.84 & 5.61 \\
$4-5$ & 51.03 & 11.42 & 2.59 & 8.30 & 0.98 & 0.31 & 23.60 & 7.16 & 2.61 \\
$5-6$ & 103.12 & 7.13 & 1.35 & 16.61 & 0.62 & no data & 44.03 & 4.30 & 1.20 \\
$6-7$ & 12.89 & 1.15 & 0.72 & 2.23 & 0.06 & no data & 9.38 & 2.37 & 0.78 \\
$7-8$ & 12.36 & 5.50 & 1.48 & 1.66 & 0.35 & no data & 10.04 & 4.66 & 1.31 \\
\hline mean & 190.49 & 46.42 & 7.67 & 28.25 & 6.37 & 2.41 & 95.36 & 33.58 & 5.07 \\
\hline
\end{tabular}

Table 2. Photosynthetic pigments for the three sites (in $\mathrm{mg} \mathrm{m}^{-2}$ ); chlorophyll $a$ data from Hamels et al. (1998)

The distribution of chlorophyll $a$ in the sediment was reported by Hamels et al. (1998). All pigments (chlorophyll $a$, chlorophyll $c$, fucoxanthin) were negatively correlated ( $\mathrm{p}<0.05$ ) with depth in the sediment. Maximum chlorophyll $a$ concentrations were recorded at Site 1; Site 2 had intermediate values and Site 3 had the lowest values. For the three sites, the bulk of the algal pigments was present in the top $2 \mathrm{~cm}$ of the sediment. For Site 2 and 3 pigment concentrations decreased gradually with depth whereas for Site 1 a more distorted depth pattern was recorded (Hamels et al. 1998). 


\section{Materials and methods}

In June 1996, the three sites (at km scale) were sampled during low water at daytime (sediments were exposed to air). At each of the three sites, 5 cores ( $3.6 \mathrm{~cm}$ diameter) were taken at $10 \mathrm{~m}$ intervals. The samples were divided into 12 horizontal slices $(0-0.5 \mathrm{~cm}, 0.5-1$ $\mathrm{cm}, 1-1.5 \mathrm{~cm}, 1.5-2 \mathrm{~cm}, 2-3 \mathrm{~cm}, 3-4 \mathrm{~cm}, 4-5 \mathrm{~cm}, 5-6 \mathrm{~cm}, 6-8 \mathrm{~cm}, 8-10 \mathrm{~cm}, 10-15 \mathrm{~cm}, 15-20$ $\mathrm{cm})$ immediately after sampling and fixed in a hot, neutral $\left(70^{\circ} \mathrm{C}\right) 4 \%$ formaldehyde solution. Meiofaunal organisms retained on a $38 \mu \mathrm{m}$ sieve were extracted from the sediment by centrifugation with Ludox (density 1.18) (Heip et al. 1985). All macrobenthos were excluded by a $1 \mathrm{~mm}$ sieve. For each slice, all nematodes were counted after staining with Rose Bengal and 120 nematodes were picked out randomly and mounted on Cobb slides for identification to species level. The nematodes were grouped into 4 feeding guilds, according to the feeding type classification of Wieser (1953).

Samples for the pigment analyses were taken from contiguous cores for meiofauna samples. Particle size distribution was determined by laser diffraction using a Malvern particle sizer. Analytical techniques for determination of the pigment content were described in Hamels et al. (1998), for the organic carbon content in Herman et al. (2000).

Horizontal and vertical patterns in nematode abundance and community composition were analysed using ordination techniques from the PC-ORD for Windows package (version 4.20, McCune \& Mefford 1999). Through ordination samples are ordered along axes according to their resemblances. A detrended correspondence analysis (DCA) was applied on vertically integrated densities (summation of all depth layers) to test the variability between replicates. Subsequently, another DCA was used to assess total community variability based on nontransformed relative abundances. Species rarer than $\mathrm{F}_{\max } / 5\left(\mathrm{~F}_{\max }\right.$ is the frequency of the commonest species) down-weighted in proportion to their frequency. Nematode diversity was expressed as Hill indices $\mathrm{N}_{0}$ and $\mathrm{N}_{1}$ (Hill 1973). In order to test for significant differences in depth integrated (total sediment column) density and diversity between the three sites, the non-parametric Kruskal-Wallis analysis by rank and pairwise multiple comparison tests were used (Conover 1971). If assumptions were met, a univariate two-way analysis of variance (ANOVA) was used to test for significant differences in depth distribution between the three sites. A 'split-plot' design was constructed with replicates nested within 'site', however, not within 'depth', following Steyaert et al. (2001). All data were $\log (\mathrm{x}+1)$ transformed prior to 
analysis. Non-parametric Spearman rank correlation coefficients were calculated to determine relationships between diversity and environmental variables along a depth gradient.

\section{Results}

\section{Density and species composition}

Horizontal. A significant difference in total (depth-integrated) nematode densities ( $\mathrm{p} \leq 0.05)$ was found when comparing all three sites (Table 3). A post hoc multiple comparison revealed only significant differences between Site 1 and 2 .

\begin{tabular}{|l|c|c|c|}
\hline & Site 1 & Site 2 & Site 3 \\
\hline Total number of species & 52 & 41 & 54 \\
Nematodes density & $2990 \pm 818$ & $1560 \pm 699$ & $2090 \pm 666$ \\
$N_{0}$ diversity & $28.4 \pm 3.58$ & $25.2 \pm 1.92$ & $32.0 \pm 2.90$ \\
$N_{1}$ diversity & $9.1 \pm 0.94$ & $8.0 \pm 2.15$ & $8.6 \pm 0.77$ \\
\hline
\end{tabular}

Table 3. Total number of species, vertically integrated nematode density (ind. $10 \mathrm{~cm}^{-2}$ ) and vertically integrated nematode diversity for the three sites (mean values from 5 samples \pm standard deviation)

Highest abundances were recorded at Site 1, lowest abundances at Site 2 and intermediate abundances at Site 3. In addition, detrended correspondance analysis, based on species densities of bulk samples, separated all three sites and illustrated the high similarity between the five replicates of each site (Fig. 2). Eigenvalues were 0.71 and 0.05 for respectively the first and second axes.

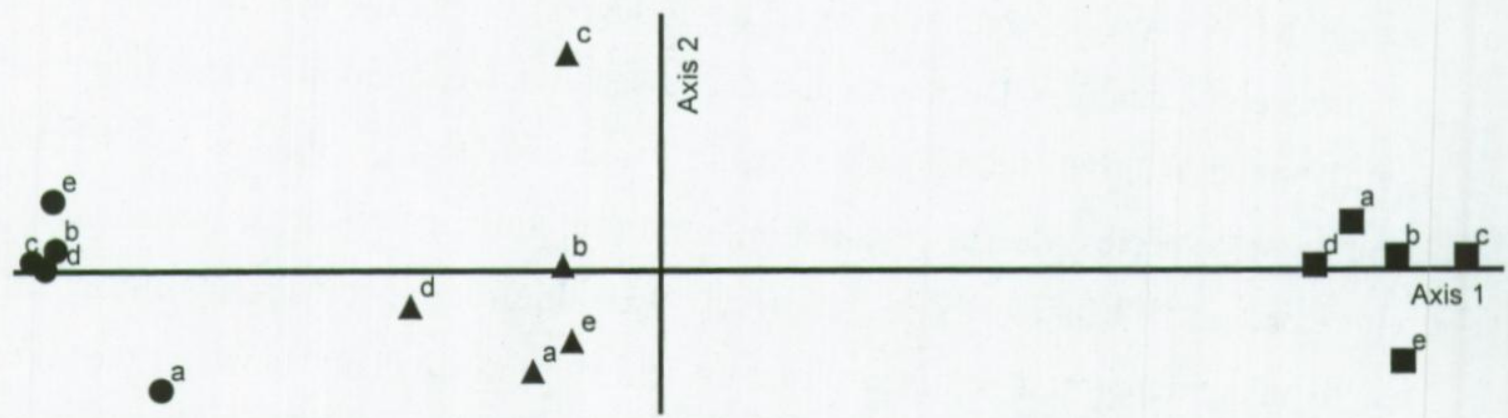

Figure 2. Results of Detrended Correspondence Analysis axes 1 and 2, based on species abundances (Site 1 triangles, Site 2 squares, Site 3 circles, letters represent replicates)

Total species number was in the same range at all three sites. In total, 76 nematode species were identified: 52 at Site 1, 41 at Site 2 and 54 at Site 3. Site 1 had 11 species exclusive to that site while Site 2 and 3 had respectively 9 and 17 unique species. Average relative abundances for the dominant species are shown in Table 4. 


\begin{tabular}{|l|c|ccc|}
\hline & Feeding type & Site 1 & Site 2 & Site 3 \\
\hline Theristus blandicor & B & 20.1 & 0.6 & 30.5 \\
Viscosia viscosa & 2B & 9.5 & 15.3 & 4.0 \\
Ascolaimus elongatus & 1B & 26.0 & 2.6 & 3.1 \\
Eleutherolaimus amasi & 1B & 14.4 & 0.2 & 3.9 \\
Theristus pertenuis & 1B & 5.2 & 0.1 & 5.6 \\
Microlaimus marinus & 2A & 2.7 & & 2.7 \\
Trefusia helgolandica & 1A & 1.0 & & 2.3 \\
Enoploides longispiculosus & 2B & 0.5 & 0.1 & 30.1 \\
Daptonema riemanni & 1B & 0.1 & 0.2 & 3.9 \\
Microlaimus acinaces & 2A & 0.3 & & 2.2 \\
Tripyloides gracilis & 1B & 2.9 & 40.1 & 0.2 \\
Ptycholaimellus ponticus & 2B & 0.3 & 12.4 & 0.1 \\
Daptonema tenuispiculum & 1B & 0.8 & 9.7 & \\
Calyptronema maxweberi & 2B & 1.6 & 2.8 & 0.1 \\
Theristus acer & 1B & 0.1 & 3.1 & 0.1 \\
Sabatieria pulchra & 1B & 1.4 & 0.9 & \\
\hline
\end{tabular}

Table 4. Relative abundances and feeding type (Wieser 1953) of the dominant species (values in bold are the dominant species per site)

Nematode species were termed dominant when present in at least 25 of the 180 slices or in at least 7 slices with a minimum relative abundance of $40 \%$ (restrictions based on practical considerations). Based on the dominant species, three different species assemblages could be recognised at the three sites: Theristus blandicor, Ascolaimus elongatus and Eleutherolaimus amasi were most dominant in Site 1 (60.5\% of total community); Tripyloides gracilis, Viscosia viscosa and Ptycholaimellus ponticus in Site 2 (67.8\%); T. blandicor and Enoploides longispiculosus in Site $3(60.6 \%)$.

Vertical profiles. ANOVA 'split-plot' analysis (Table 5) showed significant differences in depth distribution of total nematode densities (Fig. 3) and of densities for each dominant species (Fig. $4,5,6)$ among the three sites. Nematode density at Site 1 (Fig. 3) was highest at the sediment surface and decreased gradually with depth. The maximum density of each of the dominant species at Site 1 occurs at different depth layers (Fig. 4), which indicates a gradual shifting of the nematode community with depth in the sediment at Site 1 . Maximum abundance of $V$. viscosa was found in the upper sediment layer $(0-0.5 \mathrm{~cm})$. Ascolaimus elongates, E. amasi and Theristus pertenuis showed peak abundance at 0.5 to $1 \mathrm{~cm}$ depth; Microlaimus marinus and $T$. helgolandica at intermediate sediment depths (respectively $2-3 ; 6-8 \mathrm{~cm}$ ), and finally $T$. blandicor at 5 to $10 \mathrm{~cm}$ depth in the sediment (Fig. 4). 


\begin{tabular}{|l|rl|rl|rl|}
\hline & \multicolumn{2}{|c|}{ Site } & \multicolumn{2}{c|}{ Depth } & \multicolumn{2}{c|}{ Site $\times$ Depth } \\
\hline Nematode community & \multicolumn{1}{|c|}{$F$} & $\mathrm{p}$ & \multicolumn{1}{|c|}{$\mathrm{F}$} & $\mathrm{p}$ & \multicolumn{1}{c|}{$\mathrm{F}$} & $\mathrm{p}$ \\
\hline Ascolaimus elongatus & 8.982 & 0.009 & 32.725 & 0.000 & 11.301 & 0.000 \\
Calyptronema maxweberi & 58.182 & 0.000 & 47.698 & 0.000 & 26.079 & 0.000 \\
Daptonema tenuispiculum & 16.247 & 0.002 & 12.628 & 0.000 & 3.637 & 0.000 \\
Eleutherolaimus amasi & 107.517 & 0.000 & 23.619 & 0.000 & 13.099 & 0.000 \\
Enoploides longispiculosus & 30.397 & 0.000 & 5.511 & 0.000 & 7.896 & 0.000 \\
Microlaimus acinaces & 147.509 & 0.000 & 34.552 & 0.000 & 18.075 & 0.000 \\
Microlaimus marinus & 20.651 & 0.001 & 3.320 & 0.005 & 2.763 & 0.001 \\
Ptycholaimellus ponticus & 57.632 & 0.000 & 8.647 & 0.000 & 8.104 & 0.000 \\
Sabatieria pulchra & 205.658 & 0.000 & 49.261 & 0.000 & 28.031 & 0.000 \\
Theristus acer & 10.489 & 0.006 & 5.446 & 0.000 & 3.431 & 0.000 \\
Theristus blandicor & 40.125 & 0.000 & 14.292 & 0.000 & 16.890 & 0.000 \\
Theristus pertenuis & 12.632 & 0.003 & 22.656 & 0.000 & 7.444 & 0.000 \\
Theristus riemanni & 41.311 & 0.000 & 6.514 & 0.000 & 5.858 & 0.000 \\
Trefusia helgolandica & 9.746 & 0.007 & 5.386 & 0.000 & 4.449 & 0.000 \\
Tripyloides gracilis & 9.802 & 0.007 & 8.781 & 0.000 & 2.823 & 0.000 \\
Viscosia viscosa & 23.913 & 0.000 & 49.971 & 0.000 & 15.236 & 0.000 \\
& 17.188 & 0.001 & 40.367 & 0.000 & 7.210 & 0.000 \\
No diversity & & & & & & \\
N diversity & 3.929 & 0.065 & 8.664 & 0.000 & 6.227 & 0.000 \\
\hline
\end{tabular}

Table 5. Univariate ANOVA tests $(\mathrm{df}=2,11,18$ for respectively Site, Depth, Site $\times$ Depth)

At Site 2, the total nematode density was very high at the sediment surface and decreased gradually with depth (Fig. 3). Here $95 \%$ of the nematode community was confined to the upper $2 \mathrm{~cm}$ of the sediment, compared to $73 \%$ in Site 1. This steep gradient in depth is also reflected in the individual species distributions (Fig. 5). All dominant species, Calyptronema maxweberi, Daptonema tenuispiculum, P. ponticus, Theristus acer, T. gracilis and V. viscosa, showed maximum abundances in the top $1 \mathrm{~cm}$ of the sediment, except for Sabatieria pulchra and $T$. blandicor, which were more uniformly distributed downcore. For Site 3, total nematode density remained low and relatively constant until $10 \mathrm{~cm}$ depth (Fig. 3). 

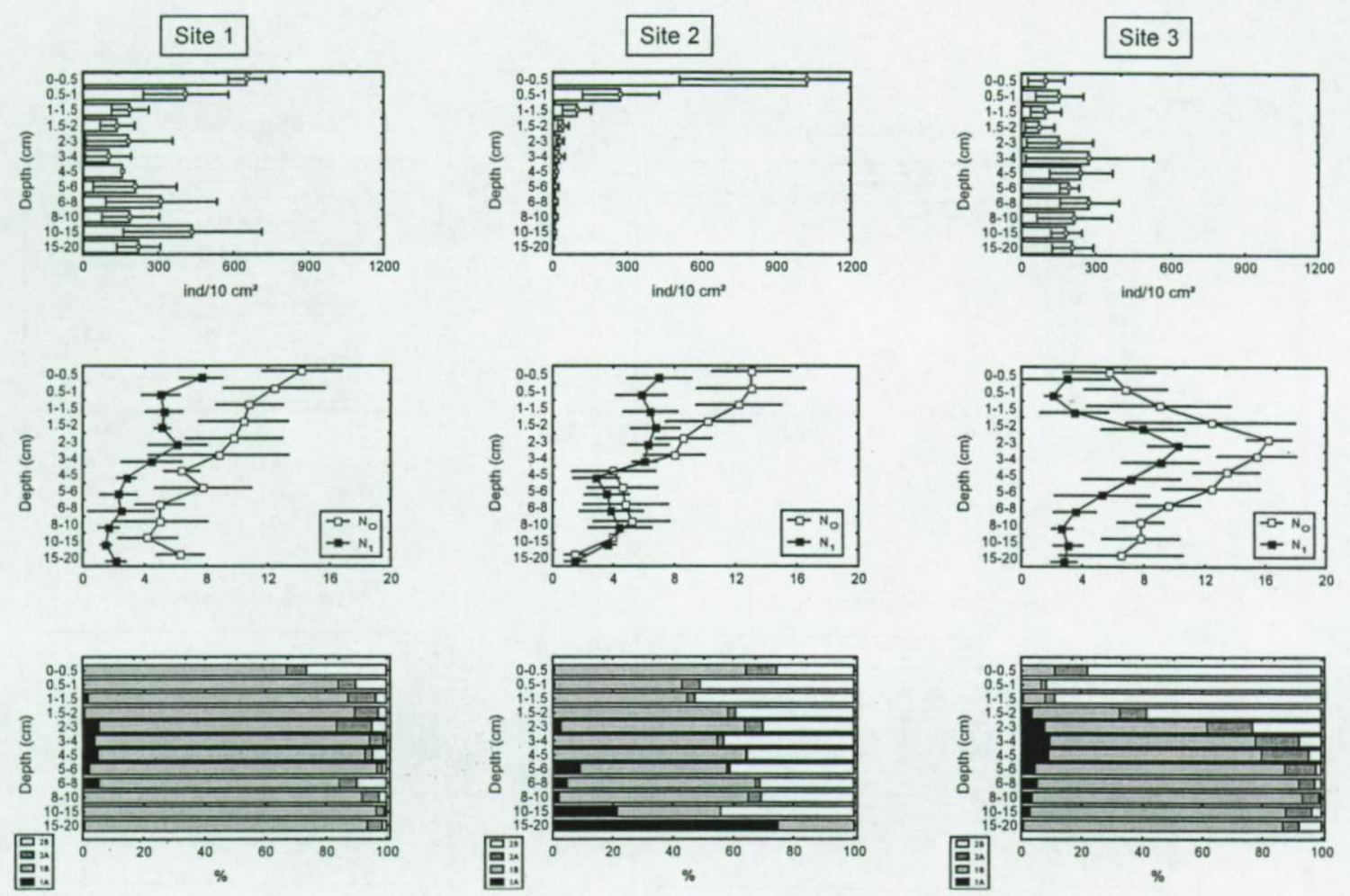

Figure 3. Nematode abundance, nematode diversity and feeding type distribution with depth

Deeper down, the abundance tended to decrease very slowly. The individual species (Fig. 6) belong to two vertically segregated species assemblages. The 'Enoploides longispiculosusassemblage' situated at the top 1.5 to $2 \mathrm{~cm}$ of Site 3 , is characterised by high abundances of $E$. longispiculosus, with a maximum abundance recorded at 0.5 to $1 \mathrm{~cm}$ depth and other species that also conform to this zonation. The 'Theristus blandicor-assemblage' is located in deeper sediment layers (from $3 \mathrm{~cm}$ onwards). The most important species here is $T$. blandicor, which has its maximum abundance at 5 to $6 \mathrm{~cm}$ depth. A similar feature is found for the less numerous species A. elongatus, E. amasi, M. marinus, M. acinaces, $T$. helgolandica, $T$. pertenuis and Theristus riemanni however species' preferences are not monotonic in this zone. Their maximum abundance is between 8 to $10 \mathrm{~cm}$. All species, except $V$. viscosa, can be attributed to one of both subcommunities. V. viscosa has highest abundance between 1.5 and 2 $\mathrm{cm}$ depth. 

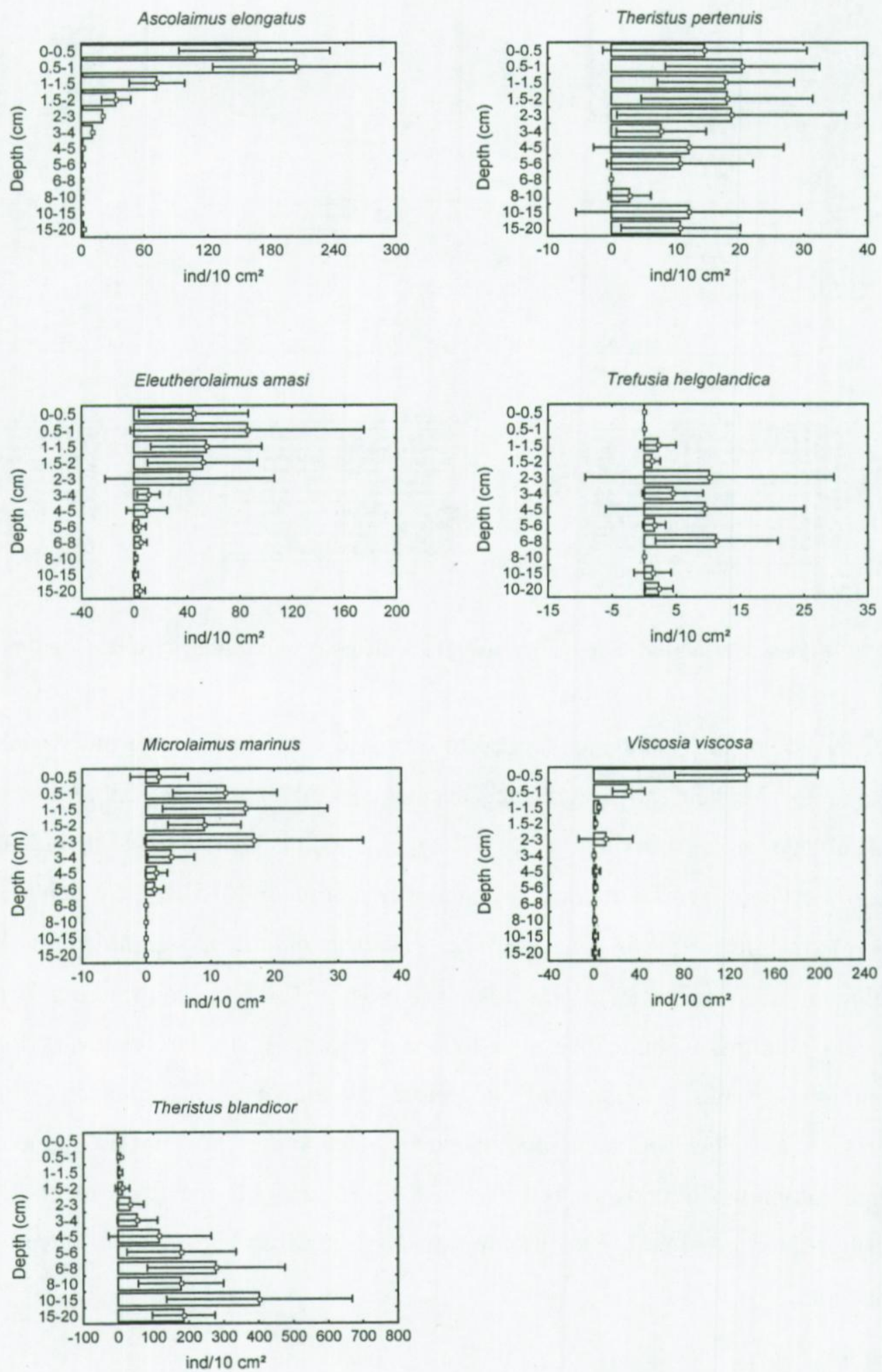

Figure 4. Depth distribution of the dominant species in Site 1 (note different width in sediment slices on $Y$ axes) 

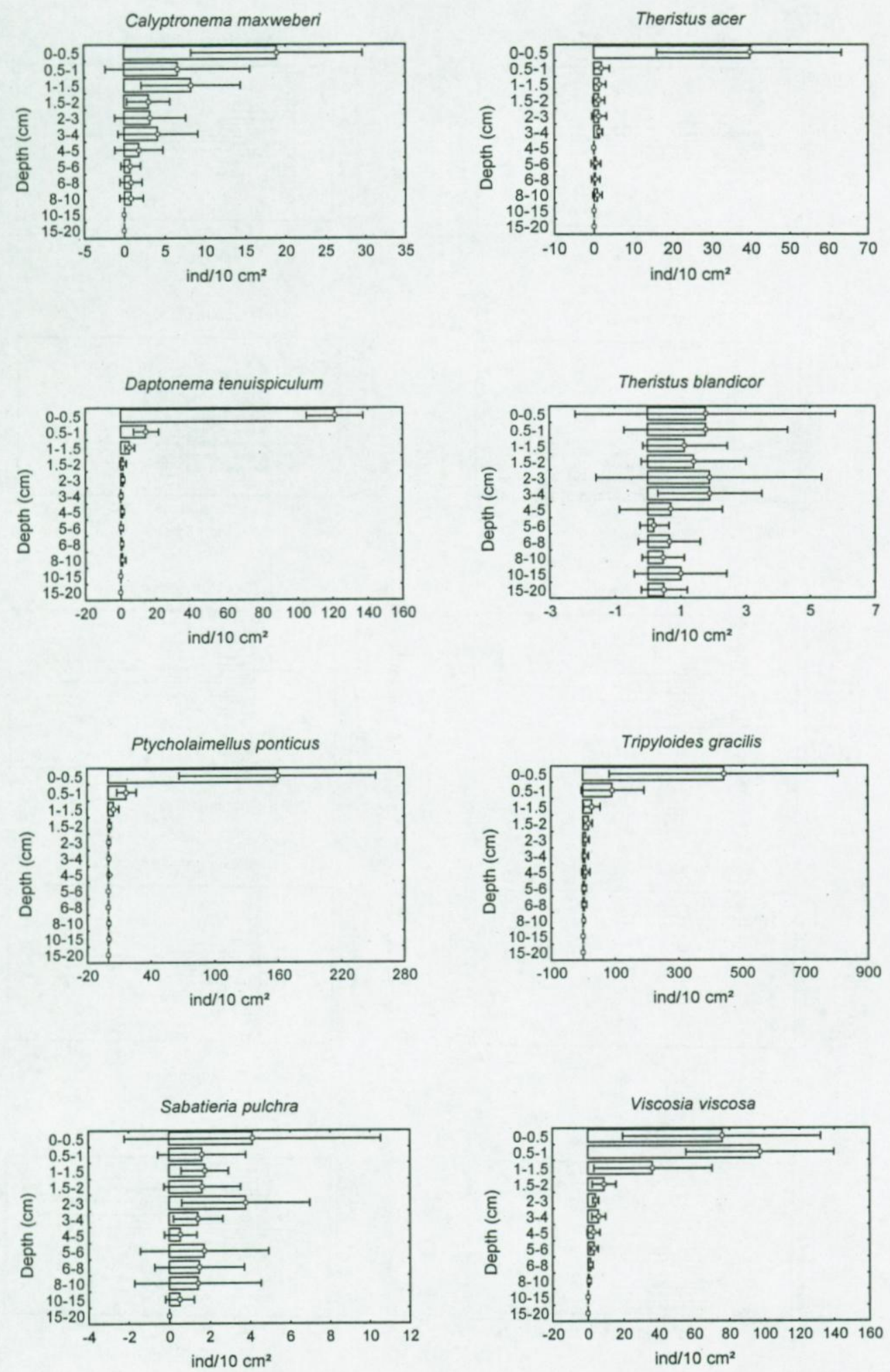

Figure 5. Depth distribution of the dominant species in Site 2 (note different width in sediment slices on $Y$ axes) 

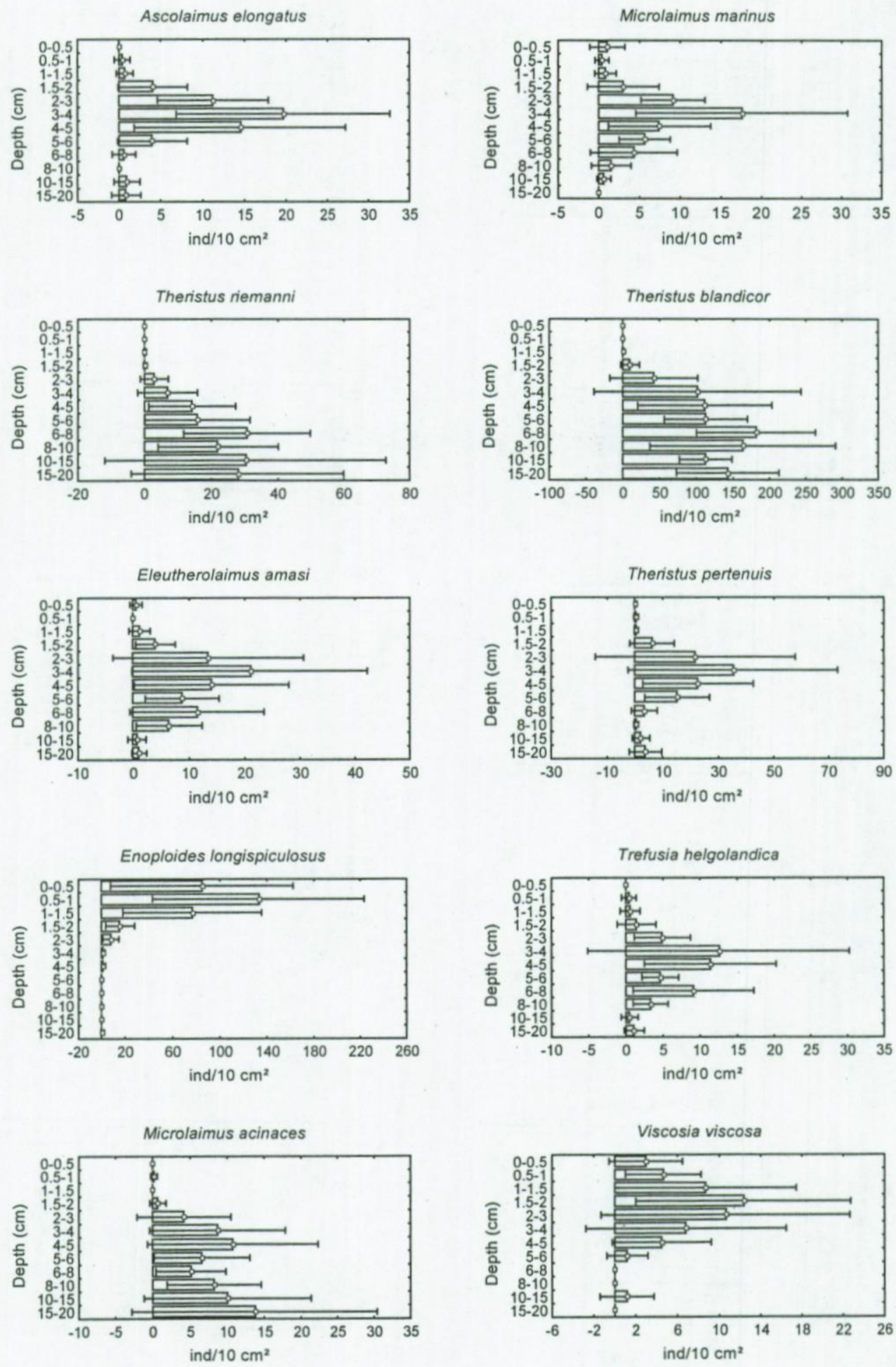

Figure 6. Depth distribution of the dominant species in Site 3 (Note different width in sediment slices on $Y$ axes) 
The existence of three different nematode assemblages at the three sites (based on dominant species and depth profiles) is confirmed by the DCA (Fig. 7). The first ordination axis has an eigenvalue of 0.787 ; the second axis has an eigenvalue of 0.42 .

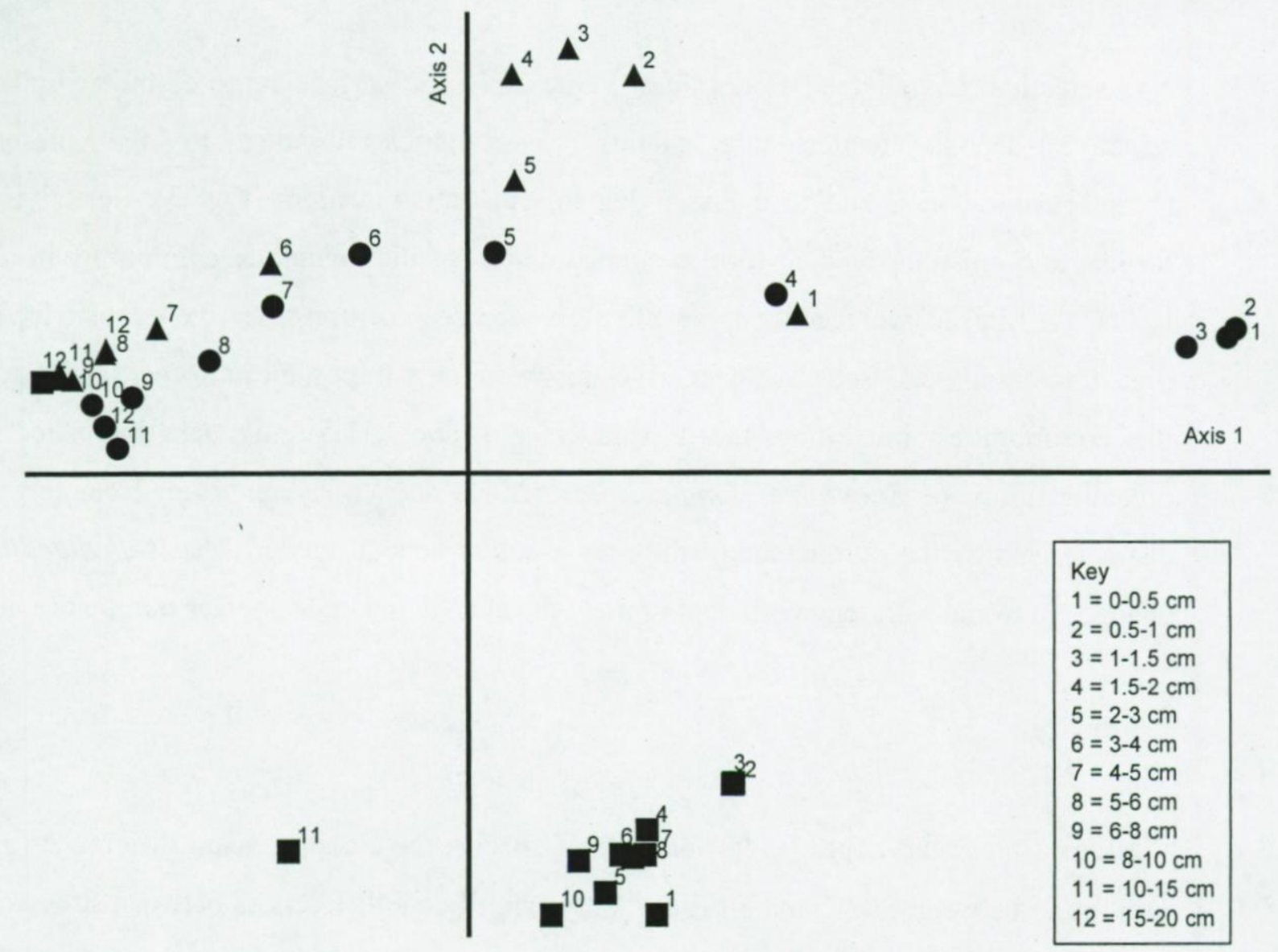

Figure 7. Results of Detrended Correspondence Analysis axes 1 and 2, based on relative abundances (Site 1 triangles, Site 2 squares, Site 3 circles)

The third axis (not depicted) has a low eigenvalue (0.098) and yields no additional information. Site 1 consists of a gradually shifting nematode community with depth. This is illustrated by the samples, which are placed along a depth gradient parallel to the first axis. It should be noticed that depth layers are plotted relatively far from each other (especially in upper depth layers). The samples of upper sediment layers of Site $3(0-0.5 ; 0.5-1 ; 1-1.5 \mathrm{~cm})$ appear separate from those of deeper sediment layers (Fig. 7), pointing again to the existence of two vertically separated subcommunities at Site 3 . The analysis further indicates that there is a similarity between deeper layers of Site 1 and 3 (beneath $2 \mathrm{~cm}$ ) and the deepest layer of Site $2(15-20 \mathrm{~cm})$, all dominated by $T$. blandicor. Site 2 is characterised by a surface nematode association that declines with depth. Vertically sectioned samples, exclusive the deepest 
sediment layer $(15-20 \mathrm{~cm})$, of Site 2 are clustered closely together and species associations seem to be similar at all depth layers.

\section{Feeding type distribution}

Non-selective deposit feeders dominated Site 1. In the surface layer of the sediment, nonselective deposit feeders are slightly less important, due to the presence of predators/omnivores, and to a lesser degree, epistratum feeders (Fig. 3). Selective deposit feeders and epistrate feeders form a small fraction of the nematode community in all depth layers. At Site 2, two feeding types are well represented: non-selective deposit feeders and predators/omnivores. Selective deposit feeders were only important in deeper layers. At Site 3, the communities inhabiting the top layers (to about $1.5 \mathrm{~cm}$ ) are dominated by the predator/omnivore Enoploides longispiculosus. The deeper layers, from $2 \mathrm{~cm}$ onwards are characterised by the dominance of the non-selective deposit feeder, Theristus blandicor. The changes in trophic strategy with depth reflect the change in single species dominance at Site 3.

\section{Diversity}

For total (integrated depth layers) number of species $\left(\mathrm{N}_{0}\right)$, a significant difference $(\mathrm{p} \leq 0.05)$ was found between Site 2 and 3 (Table 3). No significant differences between sites existed for $\mathrm{N}_{1}$ diversity. The ANOVA 'split-plot' analysis demonstrated significant differences between the three sites with respect to the variation in diversity with depth (Fig. 3, Table 5). Site 3 is clearly different from the other sites. At Site $3, \mathrm{~N}_{0}$ and $\mathrm{N}_{1}$ are low at the surface of the sediment and a maximum diversity exists at around 3 to $4 \mathrm{~cm}$ depth precisely where there is a change from a surface community to a deeper community. At Sites 1 and 2, species diversity within the sediment exhibited a different profile. At both sites, diversity was highest at the sediment surface and decreased with depth. At Site 2, there was a sharper decrease of diversity below $8 \mathrm{~cm}$ compared to Site 1 ; this can be linked to a decrease in density.

At Site 1 there was positive correlation between diversity and chlorophyll $a$, silt and a negative correlation between medium sand content (Table 6). At Site 2, diversity was positively correlated with chlorophyll $a$ and silt content. At Site 3, no significant correlations were found between diversity and any of the environmental factors. 


\begin{tabular}{|c|l|cc|cc|cc|}
\hline \multicolumn{2}{|c|}{} & \multicolumn{2}{|c|}{ Site 1 } & \multicolumn{2}{c|}{ Site 2 } & \multicolumn{2}{c|}{ Site 3 } \\
\multicolumn{2}{|c|}{} & Spearman R & $p$ & Spearman R & $p$ & Spearman R & $p$ \\
\hline Chlorophyll a & $N_{0}$ diversity & 0.865 & $\mathbf{0 . 0 0 3}$ & $\mathbf{0 . 7 2 8}$ & $\mathbf{0 . 0 2 6}$ & 0.134 & 0.731 \\
Chlorophyll a & $\mathrm{N}_{1}$ diversity & $\mathbf{0 . 7 6 2}$ & $\mathbf{0 . 0 1 7}$ & $\mathbf{0 . 7 1 1}$ & $\mathbf{0 . 0 3 2}$ & 0.251 & 0.515 \\
Organic C & $\mathrm{N}_{0}$ diversity & 0.470 & 0.202 & 0.117 & 0.764 & -0.287 & 0.454 \\
Organic C & $\mathrm{N}_{1}$ diversity & 0.371 & 0.325 & 0.134 & 0.731 & -0.226 & 0.558 \\
Silt & $\mathrm{N}_{0}$ diversity & $\mathbf{0 . 8 4 8}$ & $\mathbf{0 . 0 0 4}$ & $\mathbf{0 . 8 2 8}$ & $\mathbf{0 . 0 0 6}$ & -0.444 & 0.232 \\
Silt & $\mathrm{N}_{1}$ diversity & $\mathbf{0 . 8 9 5}$ & $\mathbf{0 . 0 0 1}$ & $\mathbf{0 . 8 6 2}$ & $\mathbf{0 . 0 0 3}$ & -0.326 & 0.391 \\
Fine sand & $\mathrm{N}_{0}$ diversity & -0.136 & 0.728 & 0.142 & 0.715 & 0.251 & 0.515 \\
Fine sand & $\mathrm{N}_{1}$ diversity & -0.134 & 0.731 & 0.126 & 0.748 & 0.318 & 0.404 \\
Medium sand & $\mathrm{N}_{0}$ diversity & $-\mathbf{0 . 7 6 3}$ & $\mathbf{0 . 0 1 7}$ & -0.176 & 0.651 & -0.159 & 0.683 \\
Medium sand & $\mathrm{N}_{1}$ diversity & $-\mathbf{0 . 8 2 8}$ & $\mathbf{0 . 0 0 6}$ & -0.192 & 0.620 & -0.226 & 0.559 \\
\hline
\end{tabular}

Table 6. Spearman Rank Order Correlations for the three sites

\section{Discussion}

\section{Nematode densities}

Previous studies addressing nematode community structure on intertidal flats, deal with diversity, distribution on different scales and production (Ott 1972, Warwick \& Price 1979, Van Es et al. 1980, Hogue \& Miller 1981, Joint et al. 1982, Pickney \& Sandulli 1990). In general, extremely high abundances of meiofauna, with nematodes always the dominant taxon, are characteristic of sheltered muddy regions of estuaries (Heip et al. 1985). This study shows a significant difference in total nematode abundance between the muddy site (Site 2, lowest densities) and Site 1, which has fine sandy sediment with high silt content and the highest nematode densities. The higher densities in Site 1, together with the higher benthic primary production (Herman et al. 2001), the higher autotrophic biomass (Hamels et al. 1998) and the larger macrobenthos stock (Herman et al. 2000), all point to a higher productivity in Site 1. This site is located at the border of the tidal flat and the open water and it is therefore exposed to stronger hydrodynamic forces compared to Site 2 (Widdows et al. 2000). As such it is likely that the input and output of fresh organic material may be larger and is the basis of the highly productive system.

Also the presence of macrofauna may affect the nematode densities although in different ways. Besides alterations of the chemical and physical properties of the sediment by macrobenthos, their effect be also linked to feeding activities (e.g. Ólafsson et al. 1993, 
Austen et al. 1998). Site 3, and in minor degree, Site 1, with higher current velocities and bottom shear stress (Van De Koppel et al. 2001) are characterised by higher densities of surface deposit feeders, while Site 2, where sedimentation rates were high (Schmidt et al. 1999 in Herman et al. 2001), is characterised by suspension feeders (Herman et al. 2001). Deposit feeders may have a predatory effect due to coincidental consumption of nematodes whilst feeding. Alternatively, the disturbance activity may stimulate microbial growth and increased sediment oxygenation, providing an increase in food and spatial resources, which in turn stimulated the nematodes (Reise 1983). Suspension feeders may stimulate nematode abundance through biodeposition of organic carbon. However it remains unclear what the macrofauna-meiofauna interaction is at the Molenplaat.

The differences in sediment characteristics, hydrodynamical conditions (reflected by current velocity and bottom shear stress) and productivity did not affect diversity of the three investigated sites and only partially total nematode abundance (only two of the three sites differed in nematode abundance). Detailed investigation of vertical depth profiles however, revealed differences that may relate to environmental factors.

Generally, the vertical distribution pattern of nematodes in silty sediments is well established: abundances are extremely high at the sediment surface or subsurface and subsequently decrease steeply with depth (for an overview, see Heip et al. 1985). This trend was evident in Site 1 and was even more pronounced in Site 2 of the Molenplaat. In the more sandy conditions of Site 3, nematode abundance remains generally lower and fluctuates greatly with depth. Oxygen penetration and the occurrence of sulphide have been coupled many times to the depth distribution of nematodes (Platt 1977, Heip et al. 1985, Giere 1993, Hendelberg \& Jensen 1993, Wetzel et al. 1995). In the sediment of Site 3, which is highly bioturbated by macrobenthos, oxygen penetration might be several centimetres in the proximity of burrows, whereas Site 2 is the least bioturbated (pers. comm. C. Barranguet). Besides a number of biotic (e.g. resource availability and distribution) and abiotic (e.g. compaction of sediment) interactions, oxygen distribution is thought to be one of the important regulating factors in explaining the obvious discrepancy in vertical distribution patterns on the Molenplaat.

\section{Community composition}

Some studies have dealt with the vertical distribution of free-living nematodes at the species level (e.g. Joint et al. 1982, Blome 1983, Warwick \& Gee 1984, Jensen 1987, Hendelberg \& 
Jensen 1993, Soetaert et al. 1994, Steyaert et al. 1999). From these studies it is clear that some nematode species show a consistent depth distribution in different areas, which suggests species-specific depth preferences. As the auto-ecological information on free-living nematodes is still very scarce, the causal factors for this depth preference are not yet completely clear. It has been suggested that the biogeochemical properties of the sediment might control the depth distribution of some species (Jensen 1981, 1987, Bouwman et al. 1984, Platt \& Lambshead 1985, Jensen \& Aagaard 1992, Steyaert et al. 1999). This might explain the surface dominated community of the silty sediment (Site 2), where oxygen penetrated only into the upper millimetres of the sediment (pers.comm. C. Barranguet).

The factors determining the vertical distribution in the fine sandy sediments may act in combination with biotic interactions. Joint et al. (1982) argued that interspecific competition gives rise to vertical niche segregation. As such, fine scale vertical stratification may play a role in allowing species with similar food requirements and feeding behaviour to co-exist in the same locality. This study supports this finding for the highly productive system of Site 1 . Here, a gradual shifting of the nematode community was recorded, as a result of the succession of maximum density peaks of dominant species with depth. This sediment was characterised by a high percentage of non-selective deposit feeders (e.g. Theristus blandicor, Ascolaimus elongatus, Eleutherolaimus amasi, Theristus pertenuis).

In the sediment of Site 3, two vertically segregated species assemblages were observed. The upper assemblage (to about $1.5 \mathrm{~cm}$ depth) was dominated by Enoploides longispiculosus. The lower assemblage (below $2 \mathrm{~cm}$ ) was dominated by $T$. blandicor and a number of less common species (Fig. 6). In earlier studies, these consistent depth profiles were also reported for some species: E. longispiculosus being a true surface-dweller (Soetaert et al. 1994) while T. blandicor, M. marinus, T. pertenuis and T. riemanni were considered as 'deep-dwelling' species (Blome 1983, Soetaert et al. 1994). The existence of these two vertically segregated assemblages on the Molenplaat is probably due to a combination of factors, of which the most important might be related to food preferences and the strong hydrodynamic regime at the site. As sediment granulometry appears to be of more importance in controlling the fauna in the upper sediment layers, the similarity of the deeper nematode communities - caused by the dominance of $T$. blandicor - at Sites 1 and 3 is particularly interesting. The environmental regime at depth seems to result in similar faunal assemblages irrespective of sediment granulometry. From its dominance in deeper sediment layers at Site 1 and 3 and the apparent lack of depth preference in Site 2 it can be concluded that $T$. blandicor persists in sediments with restrictive conditions for other nematode species. It appears that this species is capable of 
surviving anoxic conditions and is able to exploit the available food resources at depth. Such life conditions have often been described for Sabatieria species, which are typical inhabitants for deeper sediment layers of muddy intertidal and subtidal sediments (Hendelberg \& Jensen 1993, Soetaert \& Heip 1995, Steyaert et al. 1999). One Sabatieria species, S. pulchra, is also observed in this study.

\section{Diversity}

Nematode community diversity has been associated with sediment composition, oxygen, salinity, stress and organic enrichment (Giere 1993, Warwick \& Clarke 1993, Soetaert et al. 1994, Warwick et al. 1997, Austen et al. 1998, Essink \& Keidel 1998, Steyaert et al. 1999). This study documented a high nematode species richness on a small area (the Molenplaat) of approximately $2-3 \mathrm{~km}^{2}$. This high diversity may be largely the result of the heterogeneous geochemical and physical characteristics on the tidal flat. Moreover, the twofold difference in total (over the whole sediment column) number of species and averaged (over five replicates) total number of species per site, illustrates also the importance of small-scale effects. Thus, besides the mean differences, found on a large scale (between the three sites), local effects (on 10-m scale) of sedimentological characteristics are evident.

The difference in vertical profile of species richness found between Site 3 and both Site 1 and 2 , is expected as a result of the wider range of microhabitats available for meiofauna in sandy sediments (Site 3) compared to muddy sediments (Site 1 and 2) (Heip \& Decraemer 1974). Within the sediment column diversity in Site 3 is strongly variable and is only high at a depth of 2 to $5 \mathrm{~cm}$. These higher values can be explained by the occurrence of both species from the upper 'Enoploides longispiculosus-community' and the lower 'Theristus blandicorcommunity'. The lower diversity values in the uppermost layers of the sediment are related to low densities and probably result from the strong hydrodynamic regime and the tidal disturbance that prevail at the upper $2 \mathrm{~cm}$ of the sediment at Site 3 (Herman et al. 2000, Widdows et al. 2000). Therefore it is suggested that the hydrodynamic regime, which influences sediment granulometry, will predominantly affect the number of nematode species at the Molenplaat.

In many studies, correlation tests have been used to illustrate possible relationships between meiobenthos, in particular nematodes and copepods, and possible food sources (e.g. Findlay 1981, Blanchard 1990, Pickney \& Sandulli 1990, Danovaro et al. 1995, 1996 Santos et al. 
1996, Moens et al. 1999a, Steyaert et al. 1999). These studies considered, in most cases, horizontal variation. When visualising vertical distribution patterns in the sediment, correlations between individual nematode species or feeding types and biotic variables are in many cases misleading. Moreover, correlation does not imply causation. The vertical distributions of factors such as pigments, bacteria and nutrients often change with depth in the sediment. Therefore caution needs to be used when correlating depth profiles of different variables. In this study the diversity indices of Site 1 and 2, both sediments characterised by a high silt content, were positively correlated with this silt content. Such a strong positive correlation of diversity and silt content with depth has also been found in muddy sediments of the Belgian coastal zone (Steyaert et al. 1999). In general, sediment granulometry exerts an important influence on the diversity of nematode communities. On a broad, horizontal scale, coarser sediments will enhance nematode diversity by creating a broad range of microhabitats (Heip \& Decraemer 1974). Diversity on a small spatial scale, within the sediment, is inversely related to the sediment granulometry of muddy sediments. The finer the sediment becomes, the more diverse the nematode community.

\section{Conclusions}

Differences in sediment composition in association with different hydrodynamic conditions at separate sites of an estuarine intertidal flat are reflected in total nematode abundances and in species composition. The heterogeneity was much higher at $\mathrm{km}$ than at $\mathrm{m}$ scales, at which level environmental conditions seemed more consistent. Species richness in combination with equitability did not differ among the three sites, when integrated over $20 \mathrm{~cm}$ depth in the sediment. In contrast, microscale vertical profile analysis illustrated the presence of three distinctly different distribution patterns of species associations on the tidal flat: (1) in sandy sediment under strong hydrodynamic and food-stressed conditions a surface-dwelling nematode community of mainly a large predatory enoplid was observed living above a deposit feeding xyalid - microlaimid community in deeper sediment layers. These extreme environmental conditions resulted in low density and diversity. Diversity was highest at the interface between the two vertically separated communities as a result of co-existence of species from the upper and lower community. (2) In the finest sediment most nematode species were confined to the surface layers. Only a few could occasionally penetrate into 
deeper layers, resulting in a sharp decline in diversity and density with depth in the sediment. (3) At intermediate hydrodynamic and granulometric conditions there is a gradual shift from a diverse and abundant nematode community at the surface to a less diverse and less abundant one in the deeper sediment layers. The vertical changes in nematode composition resulted in a community similar to that found in the deeper sediment layers of the sandy site in terms of abundances, diversity and composition. Vertical profile analysis provides additional information over bulk sampling that is key to understanding horizontal patterns and their relation with environmental characteristics in nematode communities.

Acknowledgements. This paper benefits from financial support of ECOFLAT (The ecometabolism of an intertidal flat) and the Flemish Fund for Scientific Research FWO (3G010499). The crew of the 'Luctor', Myriam Beghyn, Johan Van de Velde and Annick Van Kenhove are acknowledged for their assistance during sampling. Myriam Beghyn and Annick Van Kenhove are also thanked for the processing of the samples. The research was supported by the BOF98-03 project from the Ghent University. This is contribution no. 356/23 of the project ECOFLAT (ENV 4-CT96-0216) to the EU ELOISE programme. 